\title{
Measurements and model calculations of the solar shortwave fluxes in snow on Summit, Greenland
}

\author{
Ingo MEIROLD-MAUTNER, * Michael LEHNING \\ WSL Swiss Federal Institute for Snow and Avalanche Research SLF, Flüelastrasse 11, CH-7260 Davos-Dorf, Switzerland \\ E-mail: lehning@slf.ch
}

\begin{abstract}
Solar shortwave (SW) radiation is a primary energy source for the snow cover. Accurate measurements of the radiation energy balance in snow are not yet available over a broad spectral band. For accurate measurements in snow, sources of errors have to be eliminated such as the temperature dependence of some sensors, investigating a disturbed snow cover or neglecting the variation of the incoming radiation. The objective of our investigation is to measure the spectral SW energy balance in and above snow and to validate a radiation transfer model against the data. In July 2002 up- and downwelling fluxes at several heights in snow were measured on Summit, Greenland, with a spectrometer sensitive in the 340-1050 nm spectral band. Changing incoming solar radiation was accounted for by using two sensor heads. The resulting data are compared to the $\delta$-Eddington model of radiative transfer in combination with Mie single-scattering calculations. Using as input the snow data obtained by weekly snow profiles, a good agreement between measured and modelled radiative fluxes was obtained when increasing the absorption in the snow grains which is justified by snow impurities. In the near-infrared a disagreement between model and measurements is obvious. This is most likely caused by non-sphericity of the snow grains.
\end{abstract}

\section{INTRODUGTION}

Light penetrating the snow cover plays a key role for many critical processes in the snow. Depending on the focus of the research, information on the availability of light in various spectral bands in snow is needed. Ultraviolet (UV) light, for instance, is the energy source for chemical reactions in snow. These processes have to be understood for a correct interpretation of ice cores and to understand the trace-gas exchange processes between snow and atmosphere. Longer wavelengths in the photosynthetic active part of the spectrum and in the near-infrared (NIR) have an effect on the ecology of snow. Vegetation underneath alpine snowpacks shows activities such as photosynthesis and photoperiodism which depend strongly on the radiation reaching them in certain spectral bands. Furthermore, algae in snow depend on the radiation available and have, due to their high absorption, a feedback on the radiation field.

Light that penetrates the snow cover also re-emerges to a large extent out of the snow cover. This fact is retained in the albedo of snow. Modelling albedo is one of the most important tasks for correct energy-balance and global climate models. A physical model of albedo results from modelling the down- and upward fluxes in snow.

Numerous studies have investigated the radiation transmission through snow. One of the first measurements with spectral detail was documented by Kuhn and Siogas (1978).

\footnotetext{
* Present address: Laboratoire d'Etude du Rayonnement et de la Matière en Astrophysicque, CNRS, 61 avenue de l'Observatoire, $75014 \quad$ Paris, France (e-mail: ingo.meirold@obspm.fr)
}

This and other measurements before 1982 are reviewed in Warren (1982). Recent measurements with spectral detail were done in the UV and visible part of the spectrum by King and Simpson (2001) (292-548 nm) and Peterson and others (2002) (290-730 nm). However, until now no measurements have been done which examined the radiation field in an undisturbed snow cover over a broad spectral band and with narrow spectral resolution. This research aims to deliver such measurements along with detailed information on the snow-cover stratigraphy. Furthermore, the performance of a current radiation transfer model for snow is validated against our measurements.

\section{METHODS}

Measurements of the radiation fluxes were taken on Summit, Greenland $\left(72^{\circ} 25^{\prime}\right.$ N, $38^{\circ} 30^{\prime}$ W; $3200 \mathrm{~m}$ a.s.l.), from 26 June to 29 July 2002. A description of the site can be found in Ohmura (2001). For our purposes, this site offered an ideal place to fulfil the requirements for the measurement set-up.

\section{Experimental set-up}

To measure the downwelling radiation fluxes in snow, a tunnel was dug with a base $3.5 \mathrm{~m}$ below the snow surface and an approximate height of $2.0 \mathrm{~m}$, resulting in $1.5 \mathrm{~m}$ of undisturbed snow for investigation (see Fig. 1). From this tunnel the radiation sensor was inserted vertically into the snow by first drilling a hole with the diameter of the sensor $(2.5 \mathrm{~cm})$ and then placing the sensor facing upwards into the hole. The length of the hole was progressively increased and 


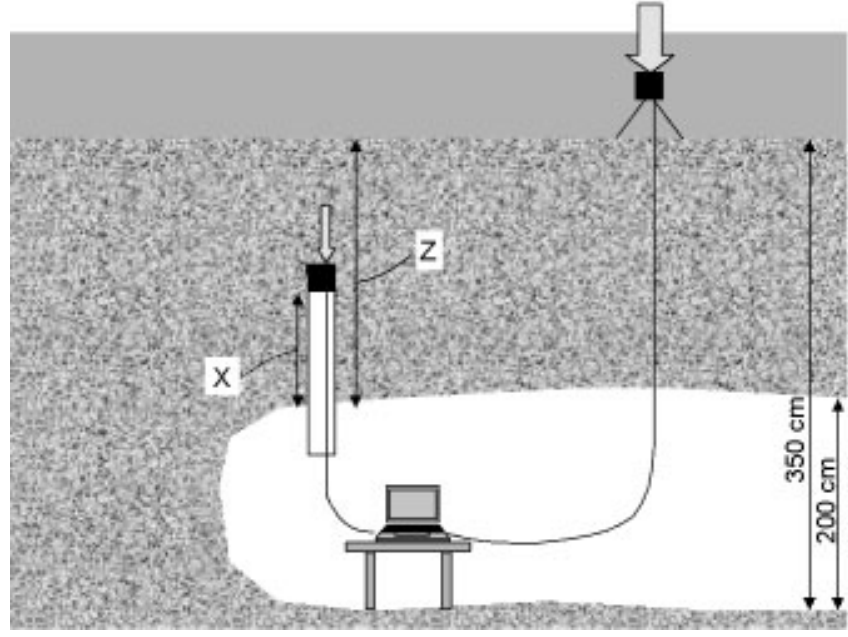

Fig. 1. Snow tunnel to measure downwelling flux. The base of the tunnel is $3.5 \mathrm{~m}$ below the surface. $1.5 \mathrm{~m}$ of undisturbed snow are available for investigation. The length of the tunnel was progressively increased to ensure undisturbed snow on each measurement day.

measurements taken until the top of the snow cover was reached.

At the same time, a reference sensor was placed on top of the snow cover to measure the incoming solar radiation. This insured that varying incoming radiation due to change in sun zenith angle and cloud cover could be accounted for.

The instrumentation consisted of an Analytical Spectral Devices FieldSpec Pro Dual visible/near-infrared (VNIR) spectrometer to which two sensor heads were connected via $4 \mathrm{~m}$ fibre-optics cables. The sensor heads have a diffuser with cosine response attached and collect radiation from one hemisphere. Data were then collected from both sensors simultaneously. The $340-1050 \mathrm{~nm}$ region of the spectrum is covered with a spectral resolution of $3 \mathrm{~nm}$ full width at halfmaximum (fwhm) and a sampling interval of $1.4 \mathrm{~nm}$.

Upwelling radiation in snow was measured by drilling a hole into the snow and inserting the sensor with the diffuser facing down. As with the measurements of the downwelling flux, the reference sensor was simultaneously measuring the incoming radiation.

In addition to the radiation measurements, weekly snow profiles to $1.5 \mathrm{~m}$ depth gave information on snow-cover stratigraphy. Snow-grain samples of the topmost layers were collected, stored in isooctane and photographed using macrophotography. These data are used to calculate the optical properties of snow and obtain parameters for a radiation transfer model.

\section{Model description}

The radiative fluxes in snow were calculated using the $\delta$ Eddington radiation transfer model. The Eddington approximation describes the intensity in the radiation transfer equation by a linear polynomial and solves the resulting two equations for constant optical properties (each layer) (see Shettle and Weinman, 1970). Snow is an extremely forwardscattering material, i.e. a beam of radiation scattered by a snow grain does not change direction appreciably. The $\delta$ function accounts for this forward scattering by approximating the phase function by a Dirac $\delta$ function (Joseph and others, 1976). This then leads to a transformation of the optical properties and hence to an improved performance of the Eddington approximation (Wiscombe and Joseph, 1977).

The $\delta$-Eddington model was applied to snow by Wiscombe and Warren (1980) and recently Simpson and others (2002). Wiscombe and Warren (1980) compared albedo measurements with this model and concluded that the model-produced albedos are too high in the visible part of the spectrum. Their subsequent paper (Warren and Wiscombe, 1980) investigates the influence of snow impurities in the model output and concludes that, with impurities added, the model represents measured albedo also in the visible range. Simpson and others (2002) present radiative flux measurements in snow from 300 to $422 \mathrm{~nm}$ and compare these with calculations using the $\delta$-Eddington model. Instead of accounting for the layering of the snow cover, they calculated the optical properties from an average grain-size for the whole snow cover and achieved good agreement between measurements and model output when decreasing the single-scattering albedo due to an additional absorber in snow.

The model requires as input the incoming radiation conditions, properties of the snow cover and the optical properties of the snow grains.

Incoming direct and diffuse radiative fluxes along with the sun zenith angle and a ground reflectivity build up the surrounding radiation boundary conditions. The reference sensor measured the total incoming irradiance, hence direct and diffuse components have to be split and are estimated following Perez and others (1991).

The layering of the snow cover comprises parameters such as number of layers and the height, density and grainsize in each layer. This information is gathered from the weekly snow profiles.

Optical properties of the snow grains are calculated for each snow layer using the Mie scattering theory for spheres. Assuming that the snow grains prevalent in Greenland are spheres, a single-scattering albedo $\omega$ (ratio of scattering to extinction), extinction efficiency $Q_{\text {ext }}$ (ratio of extinction cross-section to geometrical cross-section) and asymmetry parameter $g$ (mean cosine of scattering angle) can be calculated using for example the Mie scattering code of Wiscombe (1980). These parameters have been shown to have a ripple structure which has its origin in the electromagnetic normal modes of spheres (see Bohren and Huffman, 1998). To avoid the ripple structure, calculations are averaged over a (log-normal) size distribution of snow-grain radii centred at the respective measured radius. In natural snowpacks, grain-sizes always vary around a mean grain-size, and hence the ripple structure is smoothed out naturally.

The output of the $\delta$-Eddington model is the up and down fluxes at each layer boundary and hence also the albedo of the snow cover. Model calculations for the whole spectral range covered by the spectrometer, along with the measurements, are presented in the following section.

\section{RESULTS}

\section{Transmission measurements in snow}

The measurements on different days show similar patterns of the radiation field in snow throughout all measurement periods. The snow cover of Summit did not change very much during the period of the measurements. Only the topmost snow layers were affected by erosion and rare snow- 


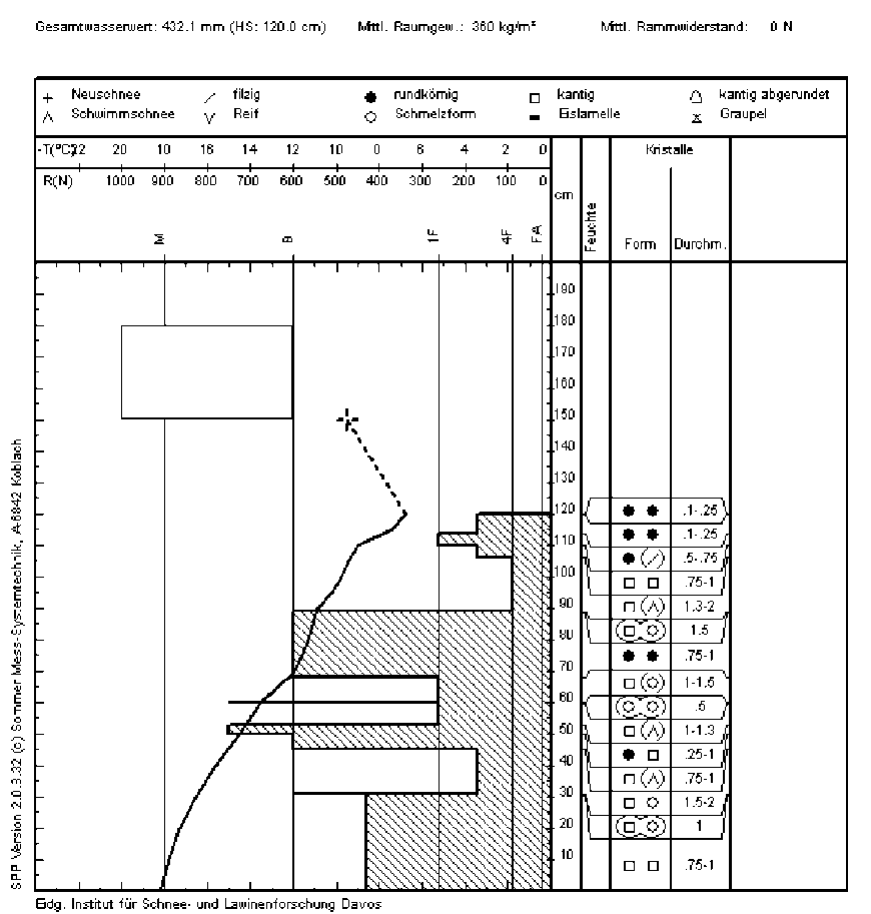

Fig. 2. Snow profile taken on 22 July 2002. The ordinate represents the depth in snow. On the right side, grain shapes along with grain diameters in $\mathrm{mm}$ are shown. The lefthand side represents the hardness of the snow, and the line gives the snow temperature.

melting events. In general, the snow structure is similar to very compact alpine seasonal snow. Grains ranged from small rounded ones with radii of $0.2 \mathrm{~mm}$ to large grains with radii of $1.5 \mathrm{~mm}$ which have undergone kinetic growth metamorphism. In the top layers densities ranged between 250 and $300 \mathrm{~kg} \mathrm{~m}^{-3}$ and after a few centimetres reached values of $350-400 \mathrm{~kg} \mathrm{~m}^{-3}$ and then remained in this range. An overview of a typical snow profile including grain-size, grain shape, snow hardness and temperature measurements is given in Figure 2.



Fig. 3. Downwelling irradiance measured on 24 Fuly 2002. The curves are the irradiances at levels 5, 10, 25, 43, 47, 57, 80,103 and $112 \mathrm{~cm}$. The two vertical lines indicate the wavelengths 660 and $730 \mathrm{~nm}$ respectively (phytochrome absorption peaks).
Table 1. Relative decrease in per cent of top level value at two selected wavelengths measured on 24 July 2002

\begin{tabular}{ccc}
\hline Depth & \multicolumn{2}{c}{ Wavelength } \\
& $660 \mathrm{~nm}$ & $730 \mathrm{~nm}$ \\
$\mathrm{~cm}$ & & \\
& & \\
5 & 7.67 & 6.04 \\
10 & 2.35 & 1.08 \\
15 & 1.42 & 0.45 \\
19 & 0.86 & 0.20 \\
25 & 0.42 & 0.07 \\
34 & 0.19 & 0.02 \\
43 & 0.05 & 0.00 \\
\end{tabular}

Note: These wavelengths are the absorption peaks of two phytochromes in plants which mediate plant processes in snow.

A typical measurement of the downwelling irradiance is represented in Figure 3. The data show irradiance curves at several layers in the snow cover. Longer wavelengths are progressively removed from the spectrum. As an example the irradiances at wavelengths 660 and $730 \mathrm{~nm}$ are presented. The relative decrease of irradiance measured on 24 July 2002 at these wavelengths is summarized in Table 1. The percentage of the surface-level value is given for each depth. It can be seen that the far red portion of the spectrum disappears faster than the red portion. Hence, snow is acting as a spectral filter for radiation.

Calculated extinction coefficients were very high for the top layer and then reached lower values, as can be seen from Figure 4. In the top few centimetres, photons entering the snow cover are very likely to re-emerge out of the snow, causing a high extinction. Further inside the snowpack the asymptotic region is reached, where irradiance decreases exponentially according to the Beer-Lambert-Bouger law (Warren, 1982).

Irradiances over the snow depths at wavelengths 350, 450, 680 and $800 \mathrm{~nm}$ are shown in Figure 5. Linear regressions to the data points are also given. The reading at the first level in snow is excluded from the regression. The $k$ values give the gradient, i.e. the extinction coefficient, of the regression lines. This figure illustrates the increasing extinction coefficient with wavelength and also indicates that the first reading in the surface layer of the snow does not fit an exponential decrease.

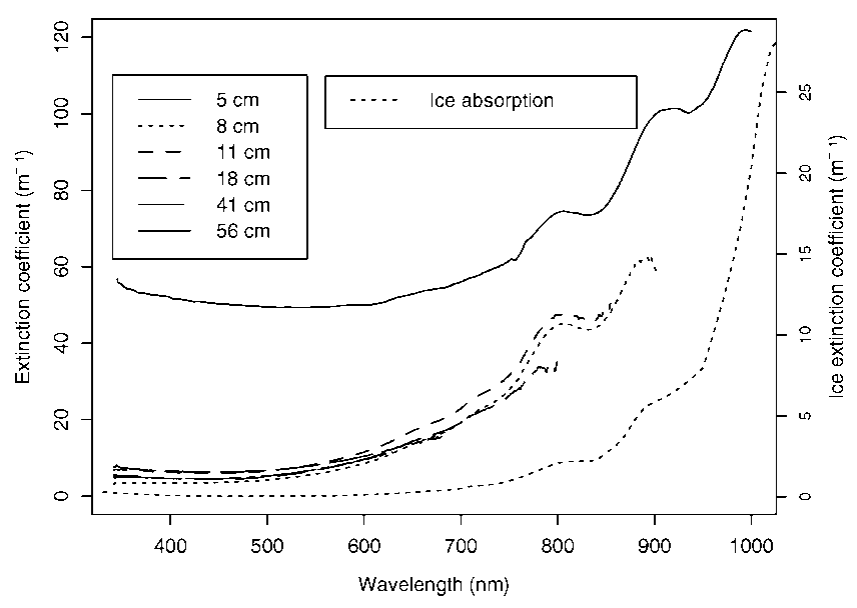

Fig. 4. Extinction coefficient derived from data measured on 23 Fuly 2002. Numbers in the legend represent levels for which the extinction is calculated, i.e. at $5,8,11,18,41$ and $56 \mathrm{~cm}$. 

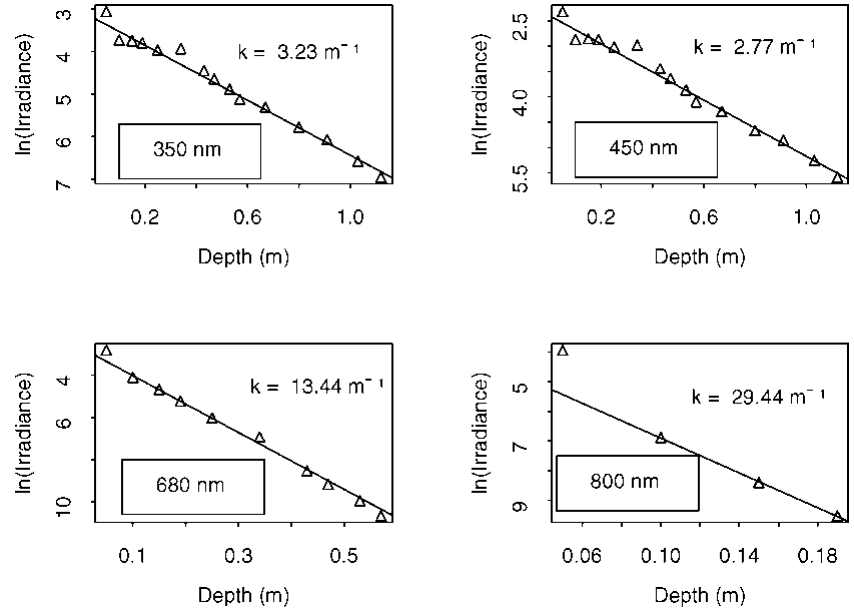

Fig. 5. Logarithm of the irradiance drawn against depth is shown as small triangles for four wavelengths. The lines are least-squares fits to the data points; the $k$ values are the gradient, i.e. the extinction coefficient, of the regression lines.

Lines are cut off in deeper layers, as the measured signal dropped under the sensitivity limit of the instrument. Increasing wavelength leads to increase in extinction because of the absorption of ice, which is also shown in the graph (data taken from Grenfell and Perovich (1981) and Perovich and Bovoni (1991)). The high-frequency variation of the extinction coefficients is a result of changes in grain-size.

\section{Comparison to the model and sensitivity studies}

Comparing the measurements with the model output shows that it is necessary, in order to obtain good agreement, to increase the absorption of the snow grains. Calculation of the single-scattering albedo $\omega$ of snow does not account for impurities, and hence the predicted value appears to be too high. Decreasing $\omega$ increases the absorption and leads to good agreement between model and measurements in the visible part of the spectrum. Longer wavelengths are less affected, since absorption is already high.

Impurity content of snow was not measured during this campaign, but it will be accounted for in further similar campaigns in the Swiss Alps. In this study, the model was fitted to match the measurements, and therefrom an impurity content deduced. Two approaches to model impurities in snow are common: the internal and the external mixture approach. In the internal mixture approach, impurities are thought of as being inside the snow crystals. The respective complex indices of refraction for ice and the impurities (e.g. soot) are averaged according to their weight fraction, and Mie calculations are done with this new index of refraction. The external approach assumes that the impurities are separate from the ice crystals and act as scatterers themselves. Optical properties for these impurities are calculated and then averaged (again by their weight fraction) with those of the ice particles. In this study we followed the external approach. Impurities were assumed to consist of soot particles with a complex index of refraction of $m=1.69+0.67 i$, a grain-size of $0.1 \mu \mathrm{m}$ and a density of $1350 \mathrm{~kg} \mathrm{~m}^{-3}$. Soot concentrations were varied until the model matched the measurements. Best agreement is found when a soot concentration of 0.3 ppmw is used for the top snow layers to $6 \mathrm{~cm}$ depth, and of $0.13 \mathrm{ppmw}$ for the layers beneath. Chýlek and others (1995) found 0.02 ppmw of black
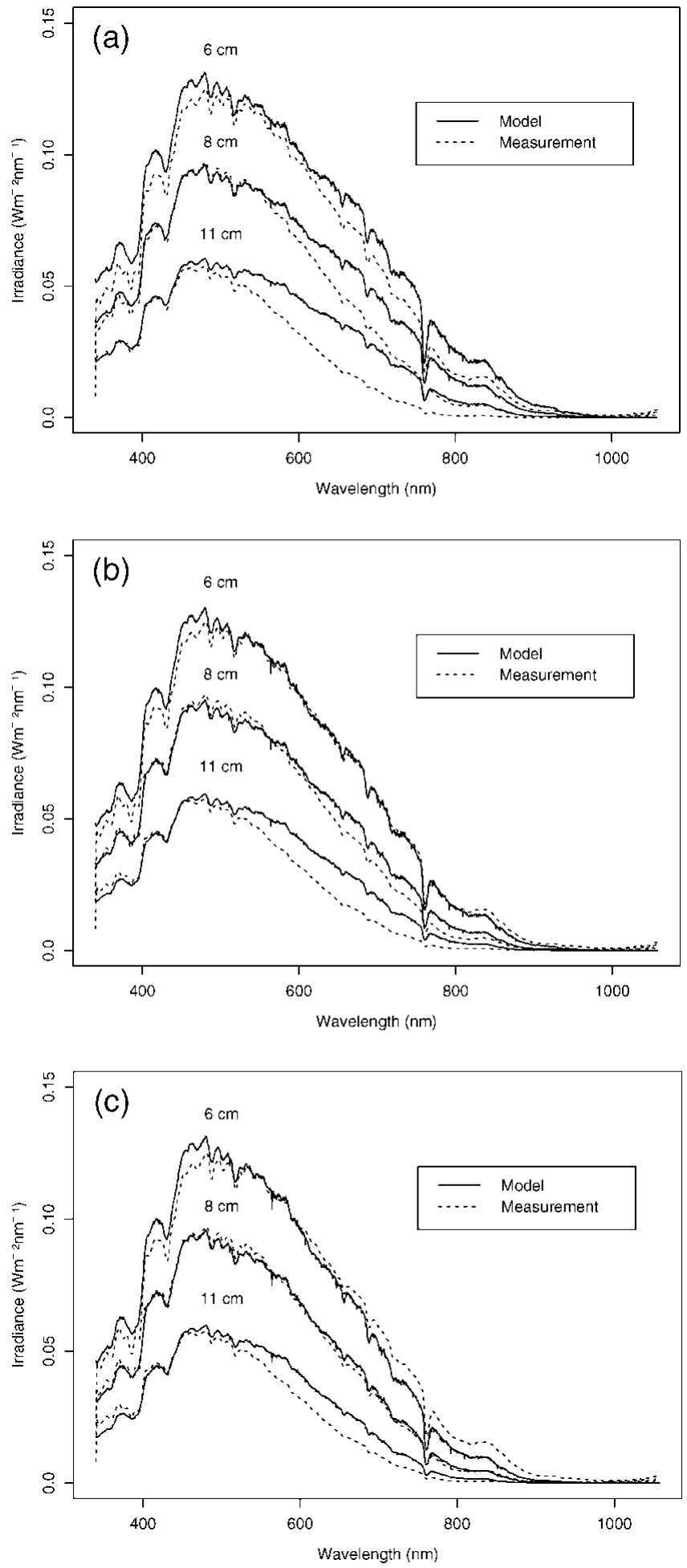

Fig. 6. Comparison of modelled and measured downward irradiance on 26 July 2002 at depths in snow at 6, 8 and $11 \mathrm{~cm}$. (a) Single-scattering albedo $\omega$ is decreased by 0.000205. (b) Grain-size scaled by 0.8, $\omega$ decreased by 0.00013. (c) Grain-size scaled by 0.8, asymmetry parameter $g$ decreased by 0.03 and $\omega$ decreased by 0.0001 .

carbon in Summit surface snow in 1989/90. In an earlier work, Warren and Wiscombe (1980) compared this model approach with albedo measurements of Grenfell and Maykut (1977) in the Arctic basin and found values around 0.3 and 0.4 ppmw.

In Figure 6a a model run along with measurements taken on 26 July 2002 is represented. Grain-sizes and densities are taken from the snow pit from 22 July 2002 which is 
shown in Figure 2. The Mie-calculated values of $\omega$ are decreased by 0.000205 for the complete wavelength range and for all layers. At wavelengths below about $500 \mathrm{~nm}$ a good agreement between model and measurement can be observed. The difference at $6 \mathrm{~cm}$ depth in this wavelength range is most likely due to uncertainties in determining the sensor depth.

An apparent disagreement between model and measurements must be observed at wavelengths above around $500 \mathrm{~nm}$. Measurements indicate a much stronger extinction than the model predicts. In this spectral region the grainsize plays a key role in correctly modelling the fluxes. In fact, techniques have been developed to retrieve grain-sizes from reflectance measurements in this spectral region (Nolin and Dozier, 2000). Larger grain-sizes increase the likelihood of photons crossing through the grains to be absorbed. Especially in the spectral region where $\omega$ is already appreciably below unity, absorption has a predominant effect in large grains.

This feature shows that it is necessary when using Mie theory, which is valid for spheres only, that an equivalent or effective grain radius (see Grenfell and Warren, 1999; Mitchell, 2002) is used, which is able to reproduce the measurements.

A first attempt is made here by scaling the measured grain-sizes by a factor of 0.8 . This causes more scattering events (thus higher extinction) to take place, and hence $\omega$ needs only be decreased by 0.00013 . The output is shown in Figure $6 \mathrm{~b}$. Now, longer wavelengths are slightly better represented but still not satisfactorily.

An additional explanation for the difference between model and measurement can possibly be found in the snow crystal shape. Approximating snow grains to be spheres may not be sufficient (even when using an effective grainsize). The phase function (which gives the average scattering angle) is especially sensitive to the shape of scatterers. The asymmetry parameter $g$ used in this study is the first approximation of the phase function and therefore a parameter depending on the grain shape. Figure $6 \mathrm{c}$ represents a model run with $g$ decreased by $0.03, \omega$ decreased by 0.0001 and grain-sizes scaled by 0.8 . A steeper decrease of irradiance at wavelengths above $500 \mathrm{~nm}$ compared to the other model runs can be observed.

Our adjustments of the parameters are made for all snow layers and for all wavelengths. This is certainly a shortcoming because effective grain-sizes have to be calculated separately for individual snow layers. Also $g$ has to be adjusted for each layer with non-spherical grain shapes separately. Nevertheless, a qualitative description of the effect of the parameter variations could be obtained.

\section{CONGLUSION}

Measurements along with modelling efforts of the radiative fluxes in Summit Greenland snow are presented in this study. The extinction coefficient calculated from the measured irradiance is very high in the top layer of the snow cover and then drops to values only altered by snow characteristics, mainly grain-size. The model used in this study is able to reproduce this feature.

Furthermore, comparisons between model and measurement indicate that modelled extinction has to be augmented by additional absorption, most likely caused by snow impurities. Estimating a soot content by fitting the model yields values of $0.13-0.3$ ppmw.

This comparison also reveals a discrepancy at wavelengths greater than $500 \mathrm{~nm}$. In this spectral region, flux extinction is strongly influenced by grain-size and probably grain shape. The influence of both parameters is examined qualitatively and shows a remarkable effect on the model output. Further work will be done in this direction, and a more physical approach will be followed. In particular, calculations of the optical properties of the snow grains will be made for non-spherical grains in favour of Mie-scattering calculations.

Finally, it is our intention to implement this radiation transfer model in a snow-cover model to be able to address ecological issues.

\section{ACKNOWLEDGEMENTS}

The authors would like to thank A. Ohmura from ETH Zürich who enabled us to take part in the project at Summitcamp, Greenland. We would like to thank W. Ammann, head of the Swiss Snow and Avalanche Research Institute, as well as M. Kuhn from the University of Innsbruck, Austria, for supporting this study. Veco Polar Resources provided the logistics in Greenland and especially all the facilities at Summitcamp. Financial support was provided by the Swiss National Science Foundation. The Mie scattering code used in this study is available from http://www.libradtran.org and the $\delta$-Eddington code can be retrieved from $\mathrm{ftp}$ //climate.gsfc.nasa.gov/pub/wiscombe/.

\section{REFERENCES}

Bohren, C. and D. Huffman. 1998. Absorption and scattering of light by small particles. New York, Wiley-Interscience.

Chýlek, P., B. Johnson, P. A. Damiano, K. C. Taylor and P. Clement. 1995. Biomass burning record and black carbon in the GISP2 ice core. Geophys. Res. Lett., 22(2), 89-92.

Grenfell, T. C. and G. A. Maykut. 1977. The optical properties of ice and snow in the Arctic Basin. 7. Glaciol., 18(80), 445-463.

Grenfell, T. C. and D. K. Perovich. 1981. Radiation absorption coefficients of polycrystalline ice from 400-1400 nm. F. Geophys. Res., 86(C8), 7447-7450.

Grenfell, T. C. and S. G. Warren. 1999. Representation of a nonspherical ice particle by a collection of independent spheres for scattering and absorption of radiation. 7. Geophys. Res., 104(D24), 31,697-31,709.

Joseph, J. H., W. J. Wiscombe and J. A. Weinman. 1976. The $\delta$-Eddington approximation for radiative flux transfer. F. Atmos. Sci., 33(12), 24522459.

King, D. and W. R. Simpson. 2001. Extinction of UV radiation in Arctic snow at Alert, Canada $\left(82^{\circ}\right.$ N). F. Geophys. Res., 106 (D12), 12,499-12,507.

Kuhn, M. and L. Siogas. 1978. Spectroscopic studies at McMurdo, South Pole and Siple stations during the austral summer 1977-78. Antarct. F. U.S., $13(4), 178-179$.

Mitchell, D. L. 2002. Effective diameter in radiation transfer: general definition, applications, and limitations. 7. Atmos. Sci., 59(15), 2330-2346.

Nolin, A. W. and J. Dozier. 2000. A hyperspectral method for remotely sensing the grain size of snow. Remote Sensing Environ., 74(2), 207-216.

Ohmura, A. 2001. Summit Greenland environment observatory. Nat. Inst. Polar Res. Mem., Ser. Special Issue 54, 153-159.

Perez, R., P. Ineichen, E. Maxwell, R. Seals and A. Zelenka. 1991. Dynamic models for hourly global-to-direct irradiance conversion. In International Solar Energy Society World Congress, 19-23 August 1991, Denver, Colorado. Proceedings. Vol. 1, Part II, 951-956.

Perovich, D. K. and J.W. Govoni. 1991. Absorption coefficients of ice from 250 to $400 \mathrm{~nm}$. Geophys. Res. Lett., 18(7), 1233-1235.

Peterson, M., D. Barber and S. Green. 2002. Monte Carlo modeling and measurements of actinic flux levels in Summit, Greenland snowpack. Atmos. Environ., 36(15-16), 2545-2551.

Shettle, E. P. and J. A. Weinman. 1970. The transfer of solar irradiance through inhomogeneous turbid atmospheres evaluated by Eddington's approximation. F. Atmos. Sci., 27(7), 1048-1055. 
Simpson, W. R., M. D. King, H. J. Beine, R. E. Honrath and X. Zhou. 2002. Radiation-transfer modeling of snow-pack photochemical processes during ALERT 2000. Atmos. Environ., 36(15-16), 2663-2670.

Warren, S. G. 1982. Optical properties of snow. Rev. Geophys. Space Phys., 20(1), 67-89.

Warren, S. G. and W. J. Wiscombe. 1980. A model for the spectral albedo of snow. II. Snow containing atmospheric aerosols. 7. Atmos. Sci., 37(12) 2734-2745.
Wiscombe, W. J. 1980. Improved Mie scattering algorithms. Appl. Opt., 19(9), 1505-1509.

Wiscombe, W. J. and J. H. Joseph. 1977. The range of validity of the Eddington approximation. Icarus, 32, 362-377.

Wiscombe, W. J. and S. G. Warren. 1980. A model for the spectral albedo of snow. I. Pure snow. 7. Atmos. Sci., 37(12), 2712-2733. 